\section{P101 COVID-19 VACCINE ACCEPTANCE IN OLDER SYRIAN REFUGEES IN LEBANON: PRELIMINARY FINDINGS FROM A LONGITUDINAL SURVEY}

${ }^{1}$ Noura El Salibi*, ${ }^{2}$ Sawsan Abdulrahim, ${ }^{1}$ Maria El Haddad, ${ }^{3}$ Stephanie Bassil, ${ }^{3}$ Zeina El Khoury, ${ }^{1}$ Hala Ghattas, ${ }^{1}$ Stephen McCall. ${ }^{1}$ Centre for Research on Population and Health, Faculty of Health Sciences, American University of Beirut, Beirut, Lebanon; ${ }^{2}$ Department of Health Promotion and Community Health, Faculty of Health Sciences, American University of Beirut, Beirut, Lebanon; ${ }^{3}$ Norwegian Refugee Council, Beirut, Lebanon

10.1136/jech-2021-SSMabstracts. 187

Background Understanding and overcoming barriers to vaccine acceptance is essential to achieve COVID-19 herd immunity worldwide.This is paramount for displaced populations who are disproportionately affected by underlying disease syndemics.Studies on COVID-19 vaccine acceptance among immigrants and refugees are limited.This study aimed to determine the prevalence and factors associated with COVID-19 vaccine refusal in older Syrian refugee beneficiaries of a humanitarian organization in Lebanon.

Methods The findings come from an ongoing rotating 4-wave panel study aiming to track older Syrian refugees' vulnerability to COVID-19. The sampling frame was a beneficiaries list of a humanitarian organization in Lebanon and included a probability sample of Syrian refugee households with at least one adult aged 50 years or older. 3,838 individuals agreed to participate in a phone survey. The present findings are limited to a sample of 1,037 from the first panel who were interviewed between January-February, 2021. Intention to vaccinate against COVID-19 was assessed through unadjusted logistic regression models to examine associations between COVID-19 vaccine refusal and socio-demographic factors, adherence practices, and vaccine and COVID-19 related perceptions using Stata/ SE13.1.

Results Out 1,037 beneficiaries, 29\% (95\%CI:0.26-0.32) reported no intention to vaccinate and 5.2\% didn't know. Among those not willing to vaccinate, the reasons were: newness of the vaccine $(35 \%)$; preference to maintain precaution measures (21\%); belief that COVID-19 vaccine is not essential (21\%); and other reasons (23\%). COVID-19 vaccine refusal was statistically significantly higher outside informal tented settlements (ITS) than inside ITS (Odds ratio (OR):1.36; 95\% CI:1.03-1.81). The odds of vaccine refusal were six and seven times higher among older Syrian refugees who did not agree that vaccines are safe (OR:5.97; 95\%CI:4.03-8.84) or effective (OR:6.80; 95\%CI:4.44-10.42) than those who agreed. Refugees aged 70 years and older and those reporting chronic conditions did not significantly differ from younger participants or those without chronic conditions in their vaccine acceptance. Self-reported adherence to COVID-19 public health measures and perceptions of susceptibility to and severity of COVID-19 were not associated with vaccine acceptance.

Conclusion This study highlights important directions towards enhancing vaccine acceptance among Syrian refugees to reach herd immunity and ensure equitable vaccination. Practical approaches to increase vaccine acceptance include disseminating accurate, accessible, and culturally appropriate information about vaccine safety and effectiveness. Whilst this large study was representative of the beneficiaries of a humanitarian organization and not of all Syrian refugees in Lebanon, it is the first to present evidence on vaccine acceptance among refugees.

\section{P102 \\ FACILITATORS AND BARRIERS TO COVID-19 VACCINE UPTAKE IN BAME GROUPS IN PRIMARY CARE: A QUALITATIVE STUDY}

${ }^{1}$ Lucia Magee*, ${ }^{1}$ Mohammad Razai, ${ }^{1}$ Roaa Al-bedaery, ${ }^{1}$ Felicity Knights, ${ }^{2}$ Douglas GJ Mckechnie. 'Population Health Research Institute, St George's, University of London, London, UK; ${ }^{2}$ Department of Primary Care and Population Health, University College London, London, UK

\subsection{6/jech-2021-SSMabstracts. 188}

Background Covid-19 vaccination is an effective strategy to reduce the spread of infection and achieve herd immunity. However, evidence suggests that both vaccine uptake and intention to vaccinate differ amongst population groups. Vaccine hesitancy is highest amongst specific ethnic minority groups. There is currently no qualitative study of the barriers and facilitators to covid-19 vaccine uptake in BAME groups in the UK primary care.

Methods We aim to conduct in-depth telephone interviews using semi-structured, open-ended questions about covid-19 vaccination in patients from South Asian (Bangladeshi/Pakistani) and Black African/African-Caribbean ethnicities in primary care in March 2021. Patients will be recruited using purposive sampling in 5 socially and ethnically diverse general practices in London. Interviews will be transcribed verbatim and subjected to thematic analysis. Data on age, sex, occupation, co-morbidities, previous vaccination status, geographical location, country of birth, education level will be also be obtained. Patients will be selected through EMIS search. All adults over 18 who are eligible for covid-19 vaccination regardless of priority status and can consent will be included in the study. Questions will relate to desire to take the vaccine, barriers and potential factors that would change their view and decision-making.

Results We hypothesise that covid-19 vaccine hesitancy will be associated with deprivation, lower educational attainment, residential segregation, previous negative healthcare experiences, and poor trust of healthcare services. Other barriers and potential solutions will be explored in depth during the interview.

Conclusion Covid-19 has had a disproportionate impact on ethnic minority groups with much higher mortality, and cases and hospitalisation rates compared to the White populations. Vaccination is an effective strategy in mitigating the risk. We need to understand the factors that cause vaccine reluctance, hesitancy and refusal, and how to facilitate engagement with vaccination programmes. This primary-care based study could help plan targeted public health campaigns to increase covid19 vaccine uptake.

\section{P103 HOW TO SUPPORT COVID-19 VACCINE UPTAKE IN THOSE THAT ARE EITHER UNDECIDED OR MORE RESISTANT TO RECEIVING A VACCINE IN ENGLAND? CONTENT ANALYSIS OF SURVEY OPEN TEXT RESPONSES}

\footnotetext{
1,2 Louis Goffe*, 1,2 Fiona Graham, 1,3Vivi Antonopoulou, ${ }^{1,3}$ Carly J Meyer, 1,2 Mei Yee Tang, ${ }^{1,2}$ Jan Lecouturier, ${ }^{1,4}$ Aikaterini Grimani, ${ }^{1,2}$ Clare Bambra, ${ }^{1,5}$ Michael P Kelly, ${ }^{1,6}$ Falko F Sniehotta. 'NIHR Policy Research Unit in Behavioural Science, Newcastle University, Newcastle upon Tyne, UK; ${ }^{2}$ Population Health Sciences Institute, Newcastle University, Newcastle upon Tyne, UK; ${ }^{3}$ Health Psychology Research Group, UCL, London, UK ${ }^{4}$ Behavioural Science Group, Warwick Business School, Coventry, UK; ${ }^{5}$ Primary Care Unit, University of Cambridge, Cambridge, UK; ${ }^{6}$ Faculty of Behavioural, University of Twente, Enschede, Netherlands
} 